\section{Análise de correspondência para avaliação do perfil de mulheres na pós-menopausa e o uso da terapia de reposição hormonal}

\author{
Correspondence analysis for the evaluation \\ of women's profile during post-menopause \\ and hormone replacement therapy
}

Renata Nunes Aranha ${ }^{1}$ Eduardo Faerstein 1 Gulnar Mendonça Azevedo ${ }^{1}$ Guilherme Werneck 1 Claudia S. Lopes 1

\section{Introdução}

1 Departamento de Epidemiologia, Instituto de Medicina Social, Universidade do Estado do Rio de Janeiro, Rio de Janeiro, Brasil.

Correspondência Renata Nunes Aranha Departamento de Epidemiologia, Instituto de Medicina Social, Universidade do Estado do Rio de Janeiro. Av. São Francisco Xavier 524, Rio de Janeiro, $R J$ 20559-900, Brasil. rearanha@uol.com.br

\begin{abstract}
This study illustrates the use of correspondence analysis to identify distinct profiles characterizing hormone replacement therapy (HRT) users and non-users, allowing the visualization of concurrent characteristics associated with this self-selection process. We analyzed cross-sectional information provided by 195 women reporting natural menopause, among 2,240 participants in the Pro-Saude Study (Rio de Janeiro, Brazil). Non-users had an unfavorable profile, including greater weight gain during adult life, older age, later menopause, and less schooling. This analytic tool should be used more extensively in public health research, in exploratory analyses of the relations among a large number of variables in key populations.
\end{abstract}

Hormone Replacement Therapy; Selection Bias; Longitudinal Studies
No Brasil, como em um número crescente de países do mundo, as mulheres já vivem, em média, quase um terço das suas vidas na fase pós-menopausa. Neste contexto, o debate sobre os possíveis benefícios e efeitos indesejáveis da terapia de reposição hormonal (TRH) sobre a saúde e a qualidade de vida assumiu grande relevância em anos recentes.

A TRH exerce benefícios evidentes sobre as repercussões clínicas precoces da menopausa (como a instabilidade vasomotora e a hipotrofia da mucosa vaginal), assim como na prevenção da osteoporose. Além disso, os recentes resultados do ensaio clínico americano - Women's Health Initiative - corroboraram evidências anteriores de associações do uso de TRH com a diminuição do risco de câncer de cólon e reto, e com aumento do risco de tromboembolismo pulmonar e de câncer de mama 1,2.

O debate principal, entretanto, diz respeito aos resultados relacionados ao risco cardiovascular. Estudos observacionais vinham sugerindo efeitos benéficos 3 , em contraste com ensaios clínicos que relataram aumento do risco de eventos secundários de coronariopatia 4 e mais recentemente, em relação a seus eventos primários 2,5,6. Essa última publicação vem forçando a modificação na indicação desta terapia, restringindo sua utilização. Do ponto de vista de saúde pública, a principal motivação 
do uso da TRH em larga escala vinha sendo a redução do risco de morrer por doenças cardiovasculares entre mulheres acima de cinqüenta anos, e tal indicação não mais se justifica.

Segundo alguns autores 7,8 , a redução do risco cardiovascular verificada nos estudos observacionais poderia ter sido motivada por um viés de seleção. Assim, existiriam, previamente ao início da terapia, diferentes características das mulheres que seriam determinantes da sua futura utilização da TRH. Tais características, por sua vez, estariam associadas a menor risco de doença cardiovascular. Em conseqüência, esse diferente perfil das mulheres usuárias da TRH em comparação com o das não-usuárias, resultariam em associação, de natureza nãocausal, entre o uso da TRH e a melhor saúde cardiovascular 9,10,11,12,13,14,15.

Os estudos sobre o perfil das mulheres que optam pelo uso da TRH, na sua maioria, foram realizados em populações norte-americanas e européias. No Brasil, somente um estudo, realizado em São Paulo, avaliou as características das usuárias da TRH por meio do uso da regressão logística, porém sem focalizar nos perfis anteriores à menopausa 16.

Para elucidar a complexa rede de características individuais que podem se relacionar ao consumo da TRH é útil avaliar a ocorrência conjunta dessas características. A análise de correspondência é uma técnica estatística que oferece essa visão global dos dados, explorando as inter-relações de um grande número de variáveis, de forma a discernir perfis entre populações 17. Neste estudo, esta ferramenta analítica é utilizada para identificar, em uma população de funcionárias públicas residentes no Rio de Janeiro, um conjunto de características sócio-econômicas e de condições de saúde anteriores à menopausa, que reflitam possíveis perfis diferenciados de usuárias e não-usuárias da terapia de reposição hormonal.

\section{Metodologia}

Esta investigação se baseia em dados seccionais de uma investigação longitudinal - o Estudo Pró-Saúde -, que investiga determinantes sociais de hábitos, comportamentos e eventos relacionados à saúde em população de funcionários técnico-administrativos de uma universidade no Rio de Janeiro.

\section{População de estudo}

Foram analisadas informações referentes a 195 mulheres, entre 2.240 participantes da Fase 1 do Estudo Pró-Saúde, que preencheram os seguintes critérios de inclusão: estar no período pós-menopausa com cessação da menstruação há, pelo menos, doze meses, e ter apresentado menopausa natural. Este último critério foi estabelecido a fim de garantir que as mulheres selecionadas encontrem-se, de fato, na fase pós-menopausa, uma vez que com a retirada do útero perde-se o parâmetro do cessar dos mênstruos. Assim, é possível que mulheres histerectomizadas não estejam no período do climatério e, logo, não tenham vivenciado as alterações hormonais e psíquicas típicas dessa fase. Essa opção também minimiza as diferenças entre os vários esquemas terapêuticos disponíveis. As mulheres com útero preservado necessitam utilizar o estrógeno associado ao progestágeno, o que pode ocasionar alguns efeitos indesejáveis. Tal associação interfere seletivamente na adesão e no estabelecimento dos efeitos protetores, em contraponto ao uso isolado da terapêutica que possui efeitos marcadamente mais estudados e definidos.

\section{Coleta de dados}

Para a obtenção das informações foi utilizado um questionário autopreenchível, desenvolvido pelos pesquisadores do Pró-Saúde e submetido a uma seqüência de pré-testes e estudo piloto para avaliação de sua adequação 18. Obteve-se o consentimento informado de todos os participantes. Foi realizada avaliação da confiabilidade, teste-reteste das informações obtidas sobre o tempo de menopausa, uso de TRH e motivo da menopausa, em uma amostra sistemática de funcionários da mesma universidade, não pertencentes ao quadro efetivo. Nessa avaliação, foram estimados coeficientes de confiabilidade kappa (IC95\%) para as questões supracitadas, de 0,84 (0,0-1,0), 0,91 (0,7-1,0) e $1,00(1,0-1,0)$, respectivamente. Devido ao pequeno número de mulheres pós-menopáusicas que realizaram o reteste $(n=22)$ realizou-se o procedimento de reamostragem (bootstrap) para calcular o intervalo de confiança.

\section{Definição das variáveis}

As variáveis utilizadas neste estudo refletem aspectos da posição sócio-econômica, das con- 
dições de saúde e dos cuidados com a vida reprodutiva. A variável dependente, de interesse central, corresponde ao status de uso da terapia de reposição hormonal. Assim, dois grupos foram formados: um composto pelas mulheres que relataram nunca ter utilizado a TRH $(\mathrm{n}=$ 116), e outro formado pelas respondentes que mencionaram já ter utilizado previamente a TRH e pelas que se encontravam em uso desta terapia no momento do estudo $(\mathrm{n}=79)$. A limitação de tamanho amostral obrigou-nos à agregação, em um único grupo, das ex-usuárias com as usuárias de TRH no momento do estudo; entretanto, este grupo é sabidamente mais homogêneo internamente em relação às mulheres que nunca foram usuárias 11 , já que a descontinuidade do tratamento costuma ser de cerca de $75 \%$ nos seis primeiros meses após o início da terapia 19.

A caracterização do perfil das usuárias e das não-usuárias de TRH foi feita com base em informações referentes ao período anterior ao início desta terapia. Para tal, utilizou-se o período da menopausa como ponto de referência, porque o início da TRH, na maioria dos casos, ocorre paralelamente ao período da menopausa ou próximo a ela. Foram selecionadas variáveis que tendem a preceder o uso da TRH, ou que não são, em princípio, modificadas por ela:

- Variáveis relacionadas às condições sócioeconômicas e características demográficas: nível de escolaridade (até o lo grau completo/2o grau completo/3o grau completo ou mais); união marital (vivem ou já viveram em união/ nunca viveram em união); cor da pele (branca/ não branca); idade no momento do estudo (tercis); idade da menopausa (mediana: < 48 anos/ $\geq 48$ anos); renda domiciliar per capita (tercis).

- Variáveis relacionadas à saúde: história de diagnóstico médico de hipertensão arterial sistêmica, diabetes ou dislipidemia; freqüência habitual de consulta odontológica de rotina (analisada como variável dicotômica, comportamento regular caracterizou-se pela visita ao dentista com freqüência mínima de dois anos); tabagismo (consumo de pelo menos cinco maços de cigarro durante toda a vida); carga tabágica (maços-ano: número médio de maços fumados por dia multiplicado pelo número de anos de tabagismo, dicotomizado a partir da mediana); índice de massa corporal (IMC) aos vinte anos (utilizou-se o peso informado aos vinte anos e a altura aferida para o cálculo, categorizado em $<21 / 21-25 />25 \mathrm{~kg} / \mathrm{m}^{2}$ ), ganho ponderal durante a vida adulta (diferença entre peso aferido no momento da pesquisa e peso informado aos 20 anos, categorizado em $\leq 10 / 10$ a $20 / \geq 20 \mathrm{~kg}$ ).
- Variáveis relacionadas à saúde reprodutiva: número de filhos nascidos vivos (nenhum/1-3/ $\geq 4$ ); uso anterior de anticoncepcional oral (alguma vez/nunca); realização de ligadura tubária (sim/não); planejamento do nascimento do 1o filho (sim/não); idade gestacional do início do pré-natal ( $<4$ meses $/ \geq 4$ meses).

\section{Análise estatística}

A análise de correspondência é uma técnica estatística multivariada de caráter exploratório e descritivo, utilizada para a análise de dados categóricos, que visa a analisar tabelas de contingência com um grande número de variáveis. Seus resultados oferecem interpretação similar àqueles obtidos pela análise de fatores, utilizada preferencialmente para variáveis contínuas. O programa utilizado foi uma adaptação do SPAD (Systeme Portable pour l'Ánalyse dês Donnés) 20.

Para interpretação dos resultados obtidos por meio da análise de correspondência é necessário o entendimento de algumas etapas analíticas 17. A seguir serão detalhadas estas etapas utilizando-se a Tabela 1 como exemplo ilustrativo:

\section{- Normalização}

É a padronização das freqüências relativas de forma que a contribuição de todas as células (por linha e por coluna) da tabela de contingência some $100 \%$. Para tal, inicialmente, é necessário estabelecer os perfis de linhas e colunas. Chama-se de perfil de linha a proporção representada por uma determinada célula no total daquela linha (soma de todas as células da linha). Como exemplo, na Tabela 2, a célula formada pela interseção da categoria "1o grau completo" na linha 1 e "não-usuária de TRH" na coluna 1 contém 67 mulheres. O perfil de linha é então calculado pela razão do valor da célula (67 mulheres) pelo total da linha (100 mulheres que possuem "1o grau completo"), ou seja, 0,67 (67/100). Analogamente, o perfil de coluna da mesma célula se calcula pela razão entre essas 67 mulheres e o total da coluna "não-usuária de TRH" (109 mulheres), logo 0,61 (67/109). O mesmo cálculo será realizado para o restante das células.

\section{- Matriz distância}

Esta matriz é formada pelo cálculo das distâncias entre todas as combinações de categorias da tabela tomadas dois a dois. Essa distância é definida como somatório do quadrado da diferença entre o perfil de coluna de uma categoria 
e o perfil de coluna da outra, divididos pela proporção de contribuição daquela linha no total.

$\frac{\left(P 1_{1}-P 2_{1}\right)^{2}}{L^{1}}+\frac{\left(P 1_{2}-P 2_{2}\right)^{2}}{L^{2}}+\ldots+\frac{\left(P 1_{\mathrm{n}}-P 2_{\mathrm{n}}\right)^{2}}{L^{\mathrm{n}}}$

onde $P$ é o perfil de coluna, $L$ é a contribuição da linha no total e $n$ é o número de linhas.

Seguindo o exemplo da Tabela 2, o cálculo da distância para o par das categorias "Uso" e "Não uso" da TRH pode ser expresso por:

$\frac{(0,61-0,41)^{2}}{0,53}+\frac{(0,26-0,24)^{2}}{0,25}+\ldots+\frac{(0,13-0,34)^{2}}{0,22}$

Esses passos são repetidos analogamente para o perfil de linhas, trocando-se $L$ por $C$ na equação acima. Os valores destas distâncias compõem uma matriz simétrica, de valores não negativos e que obedecem a uma distribuição do tipo qui-quadrado ponderada, já que existe uma ponderação pelo peso das linhas $(L)$ dentro da soma.

\section{- Alocação dos pontos no espaço}

Da matriz gerada anteriormente, cada linha/ coluna representa uma categoria de uma variável. A tarefa agora é alocar estes objetos no espaço preservando as relações (distâncias) entre eles, para assim obter uma melhor visualização e interpretação dos resultados.

Por meio de técnicas matemáticas, como a de escalonamento multidimensional, determi- na-se o número de dimensões (fatores) que serão utilizados para essa representação no espaço. Este processo matemático ocorre de acordo com a seguinte lógica: se há uma distância entre os pontos A e B de $2 \mathrm{~cm}$, B e C de $8 \mathrm{~cm}$ e A e C de $10 \mathrm{~cm}$, é possível utilizar uma única dimensão para representar esses pontos no espaço.

Eixo único = A--B--------C

Porém, se as três distâncias forem todas de $10 \mathrm{~cm}$, são necessárias mais dimensões para representar os mesmos pontos, e uma disposição possível para sua representação será a de um triângulo eqüilátero. Os procedimentos matemáticos realizados por técnicas computacionais definem a melhor projeção para plotar o conjunto de dados, maximizando sua visibilidade. Parte deste procedimento inclui a escolha dos eixos mais representativos. Não existe

Tabela 1

Tabela de contingência: usuárias e não-usuárias de Terapia de

Reposição Hormonal, segundo grau de escolaridade. Estudo Pró-Saúde, 1999.

\begin{tabular}{lcc}
\hline Escolaridade & $\begin{array}{c}\text { Terapia de Reposição Hormonal } \\
\text { Não uso (n) }\end{array}$ & Uso (n) \\
\hline Até 1의 grau completo & 67 & 33 \\
2o grau completo & 28 & 19 \\
3으 grau completo & 14 & 27 \\
\hline
\end{tabular}

Tabela 2

Cálculo dos perfis de linha e coluna.

\begin{tabular}{|c|c|c|c|c|c|c|}
\hline \multirow[t]{3}{*}{ Escolaridade } & \multicolumn{4}{|c|}{ Terapia de Reposição Hormonal } & \multicolumn{2}{|c|}{ Total } \\
\hline & \multicolumn{2}{|c|}{ Não Uso } & \multicolumn{2}{|c|}{ Uso } & \\
\hline & $\mathrm{n}$ & & $n$ & & $\mathrm{n}$ & \\
\hline \multirow[t]{2}{*}{ Até 1ㅇ grau completo } & 67 & $*(0,67)$ & 33 & $(0,33)$ & 100 & $(1,00)$ \\
\hline & $\star \star(0,61)$ & & $(0,41)$ & & $(0,53)$ & \\
\hline \multirow[t]{2}{*}{ 2o grau completo } & 28 & $(0,60)$ & 19 & $(0,40)$ & 47 & $(1,00)$ \\
\hline & $(0,26)$ & & $(0,24)$ & & $(0,25)$ & \\
\hline \multirow[t]{2}{*}{ 3으 grau completo ou mais } & 14 & $(0,34)$ & 27 & $(0,66)$ & 41 & $(1,00)$ \\
\hline & $(0,13)$ & & $(0,34)$ & & $(0,22)$ & \\
\hline \multirow[t]{2}{*}{ Total } & 109 & $(0,58)$ & 79 & $(0,42)$ & 188 & $(1,00)$ \\
\hline & $(1,00)$ & & $(1,00)$ & & $(1,00)$ & \\
\hline
\end{tabular}

* Perfil de linha

** Perfil de coluna 
consenso nos critérios estabelecidos para a definição do número de fatores 21 . Optou-se por aqueles que contribuem com a maior parte da explicação das informações contidas naquele conjunto de dados. Geralmente os dois primeiros fatores, responsáveis pela melhor projeção, possuem grande parte da explicação e possibilitam uma visão única em duas dimensões. Este resultado gráfico (Figura 1) está, então, pronto para interpretação. Inicialmente, avalia-se a contribuição das variáveis para cada fator retido e após esta etapa é, então, realizada a análise do gráfico produzido por esses fatores:

\section{- Análise dos fatores (eixos)}

Análise dos fatores (eixos): Para tal, observa-se em ordem decrescente a porcentagem de contribuição da explicação dos dados fornecida por cada fator, sabendo-se que o somatório dos fatores explica $100 \%$ da variância global;

Análise de cada fator: Após determinar os fatores que serão avaliados é importante conhecer as categorias das variáveis que mais contribuem na variância deste fator. Observaremos as contribuições absolutas de cada variável no lado positivo e/ou negativo do eixo, e de acordo com a categoria das variáveis que mais contribuem podemos caracterizar os fatores conceitualmente. Por exemplo, o fator pode ser mais explicado pelas variáveis que representam o nível sócio-econômico;

\section{- Análise do gráfico}

Observamos a existência de conglomerados e a proximidade das variáveis, entendendo assim os padrões de relações entre essas características. Quanto mais próximas duas variáveis no gráfico mais freqüente a sua ocorrência conjunta. O gráfico que representa dois fatores gera quatro quadrantes. Os dois fatores conjuntamente separam as características que se alocam no quadrante superior esquerdo das características que se encontram no quadrante inferior direito, e as que estão no quadrante superior direito daquelas que estão no inferior esquerdo, caracterizando grupos com perfis extremos opostos.

\section{Resultados}

Neste estudo, os dois primeiros fatores explicaram $37,0 \%$ da variabilidade global dos dados, o primeiro contribuindo com $24,7 \%$ e o segundo com $12,3 \%$; esses dois fatores foram retidos para a análise subseqüente. A análise da porcen- tagem de contribuição de cada variável na composição de cada fator evidenciou, no primeiro, uma maior influência das variáveis que reúnem informações sobre o nível sócio-econômico (por exemplo: níveis altos de escolaridade e renda domiciliar per capita no lado positivo e níveis baixos no lado oposto). As variáveis que mais influenciaram o segundo fator reúnem informações sobre o planejamento e cuidado com a vida reprodutiva (por exemplo: não viveu em união marital, não teve filhos e nunca usou TRH no lado positivo e os comportamentos opostos no lado negativo). A análise gráfica dos dois primeiros fatores (Figura 1), permitiu a identificação de quatro subgrupos. Pode-se afirmar que o gráfico separa as mulheres, investigadas neste estudo, de forma vertical segundo fatores sócio-econômicos; e de forma horizontal, segundo as características relacionadas à vida reprodutiva.

O grupo que contém as não-usuárias da $\mathrm{TRH}$, localizado no quadrante superior esquerdo, apresenta as seguintes características principais: IMC aos vinte anos superior a $25 \mathrm{~kg} / \mathrm{m}^{2}$, ganho ponderal durante a vida adulta superior a 20kg, faixa etária de 52 a 67 anos (2o e 3o tercis de idade), menopausa com 48 anos ou mais, diagnóstico médico de doença prévio à menopausa, visitam o dentista de forma irregular, detêm renda domiciliar per capita e escolaridade baixas. Predominam também as mulheres com cor da pele parda ou negra, que não planejaram o nascimento do primeiro filho, que iniciaram o pré-natal com quatro meses ou mais de gestação e, em menor grau, as que tiveram 4 ou mais filhos nascidos vivos.

O grupo que contém as usuárias da terapia de reposição hormonal está localizado no quadrante inferior direito. Predominam mulheres que fazem uso de pequena carga tabágica $(<23$ maços-ano), com nível de escolaridade médio (2o grau completo), mais jovens (idade entre 38 e 52 anos), pararam de menstruar mais precocemente (com menos de 48 anos), são brancas, aos vinte anos tinham IMC entre 21,0 e 22,9kg/ $\mathrm{m}^{2}$, apresentam ganho ponderal durante a vida adulta inferior a $10 \mathrm{~kg}$, e menor freqüência de diagnóstico médico, antes e após a menopausa, das doenças avaliadas.

Existe um grupo, localizado no quadrante inferior esquerdo da Figura 1, que se caracteriza, principalmente, pelas mulheres que vivem ou já viveram em união marital, tiveram de 1 a 3 filhos, planejaram o nascimento do 1o filho, iniciaram o pré-natal em idade gestacional adequada, fizeram uso de anticoncepcionais orais e realizaram ligadura tubária (método irreversível para contracepção), e relataram renda per 
capita entre 315 e 680 reais (2o tercil). Esse grupo não se caracteriza por ser ou não usuário de TRH, mas encontra-se mais próximo das usuárias, separado pelo fator 2 (relacionado à saúde reprodutiva) que corta horizontalmente o gráfico. No extremo do quadrante superior direito, definindo um padrão isolado e diferenciado, encontram-se as mulheres que nunca viveram em união marital, não tiveram filhos e também não utilizaram métodos contraceptivos.

\section{Discussão}

No presente estudo, comparamos algumas características prévias à menopausa das mulheres usuárias e não-usuárias da terapia de reposição hormonal. Até onde foi possível identificar na literatura entre os estudos com objetivos similares, este foi o único a utilizar a análise de correspondência. Apesar de não estabelecer a significância estatística das associações e não avaliar o efeito independente de cada característica, este método combina vantagens de métodos não lineares e de métodos multidimensionais, o que permitiu a descrição das características que ocorrem conjuntamente nesta população, e a identificação de perfis diferenciados de usuárias e não-usuárias da TRH.

Nessa população, indicadores sócio-econômicos e relacionados à saúde foram capazes de distinguir usuárias e não-usuárias da TRH. As não-usuárias apresentaram um nível inferior de escolaridade, eram menos freqüentemente brancas, eram mais velhas, apresentaram menopausa mais tardia e maior ganho ponderal durante a vida adulta. As usuárias apresentaram características opostas. A renda domiciliar per capita baixa, a irregularidade da freqüência habitual à consulta odontológica e o IMC mais elevado aos 20 anos caracterizaram somente as não-usuárias.

Apenas o menor tercil de renda per capita associou-se ao não uso da TRH, o que pode ser explicado pelo custo relativamente alto desta terapia. Estes resultados corroboram o estudo brasileiro de Pinto Neto et al. 16, onde o uso da TRH foi maior nos estratos sociais mais elevados.

Com relação à educação, as análises foram concordantes com outros trabalhos que demonstraram um maior nível educacional entre as usuárias da TRH quando comparadas às não-usuárias 9,11,14,15,16,22,23,24. Embora o nível de escolaridade apresente estreita relação com o tipo de emprego, controle sobre a saúde e acesso médico, o conjunto destas variáveis não é suficiente para explicar a forte ligação da educação com a TRH 25. Provavelmente, o me-

Figura 1

Visualização das características das usuárias e das não-usuárias da Terapia de Reposição Hormonal, por intermédio do gráfico gerado pela análise de correspondência entre os fatores 1 e 2. Estudo Pró-Saúde, 1999.

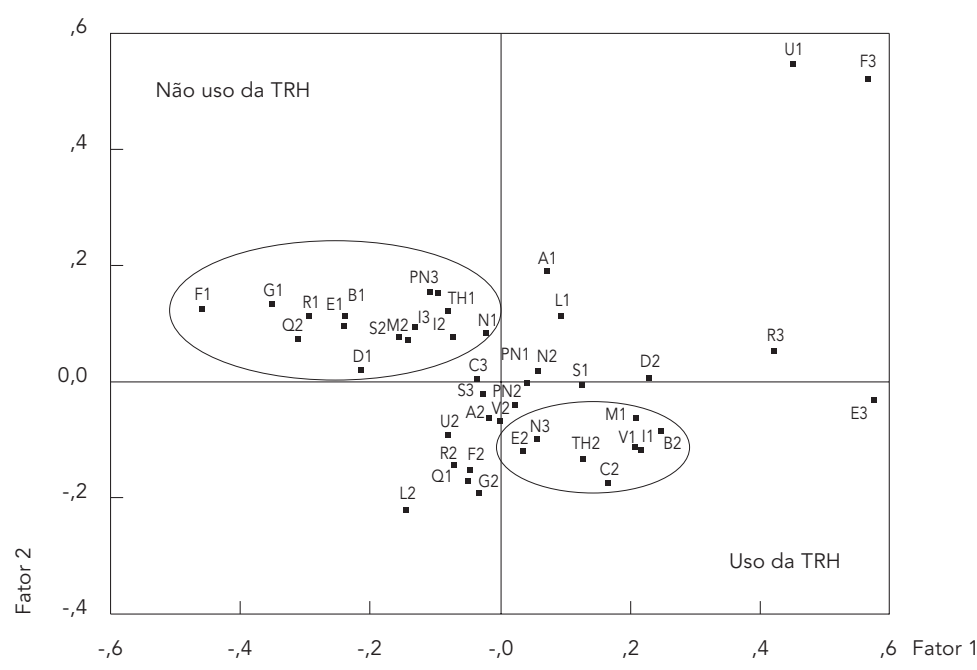

Sigla Categoria da variável

\begin{tabular}{|c|c|c|c|}
\hline A1 & $\begin{array}{l}\text { Nunca usou } \\
\text { anticoncepcional oral }\end{array}$ & M2 & $\begin{array}{l}\text { Menopausa com } 48 \text { anos } \\
\text { ou mais }\end{array}$ \\
\hline A2 & Já usou anticoncepcional oral & N1 & Nunca fumou \\
\hline B1 & Negra ou parda & N2 & Fumou antes da menopausa \\
\hline B2 & Cor branca & N3 & Fumante atual \\
\hline $\mathrm{C} 2$ & $\begin{array}{l}\text { Carga tabágica } \\
<\text { que } 23 \text { maços-ano }\end{array}$ & PN1 & $\mathrm{IMC}<21 \mathrm{~kg} / \mathrm{m}^{2}$ \\
\hline C3 & $\begin{array}{l}\text { Carga tabágica } \\
\geq \text { que } 23 \text { maços-ano }\end{array}$ & PN2 & $I M C \geq 21$ a $25 \mathrm{~kg} / \mathrm{m}^{2}$ \\
\hline D1 & Visitas irregulares ao dentista & PN3 & $\mathrm{IMC} \geq$ a $25 \mathrm{~kg} / \mathrm{m}^{2}$ \\
\hline D2 & Visitas regulares ao dentista & Q1 & $\begin{array}{l}\text { Planejou o nascimento } \\
\text { do primeiro filho }\end{array}$ \\
\hline E1 & $\begin{array}{l}\text { Escolaridade até } \\
\text { 1ㅇ grau completo }\end{array}$ & Q2 & $\begin{array}{l}\text { Não planejou o nascimento } \\
\text { do } 1 \text { o filho }\end{array}$ \\
\hline E2 & $\begin{array}{l}\text { Escolaridade - 2o grau } \\
\text { completo }\end{array}$ & $\mathrm{R} 1$ & $\begin{array}{l}\text { Renda domiciliar } \\
\text { per capita 1으 tercil }\end{array}$ \\
\hline E3 & $\begin{array}{l}\text { Escolaridade - 3o grau } \\
\text { completo ou }+\end{array}$ & $\mathrm{R} 2$ & $\begin{array}{l}\text { Renda domiciliar } \\
\text { per capita 2o tercil }\end{array}$ \\
\hline $\mathrm{F} 1$ & Filhos nascidos vivos $\geq 4$ & R3 & $\begin{array}{l}\text { Renda domiciliar } \\
\text { per capita 3o tercil }\end{array}$ \\
\hline F2 & De 1 a 3 filhos nascidos vivos & S1 & $\begin{array}{l}\text { Nenhum diagnóstico } \\
\text { médico de doença }\end{array}$ \\
\hline F3 & Não teve filhos nascidos vivos & $\mathrm{S} 2$ & $\begin{array}{l}\text { Diagnóstico médico de doença } \\
\text { prévia à menopausa }\end{array}$ \\
\hline G1 & $\begin{array}{l}\text { Idade gestacional } \geq 4 \\
\text { meses no início do pré-natal }\end{array}$ & S3 & $\begin{array}{l}\text { Diagnóstico médico de doença } \\
\text { após a menopausa }\end{array}$ \\
\hline G2 & $\begin{array}{l}\text { Idade gestacional }<4 \\
\text { meses no início do pré-natal }\end{array}$ & TH1 & $\begin{array}{l}\text { Não usuária da terapia } \\
\text { de reposição hormonal }\end{array}$ \\
\hline 11 & Faixa Etária - 38 a 52 anos & $\mathrm{TH} 2$ & $\begin{array}{l}\text { Usuária da terapia } \\
\text { de reposição hormonal }\end{array}$ \\
\hline 12 & Faixa Etária - 52 a 57 anos & U1 & Nunca viveu em união marital \\
\hline 13 & Faixa Etária - 57 a 67 anos & U2 & Já viveu ou vive em união marital \\
\hline L1 & Não fez ligadura tubária & V1 & $\begin{array}{l}\text { Ganho ponderal durante } \\
\text { a vida adulta } \leq 10 \mathrm{~kg}\end{array}$ \\
\hline L2 & Fez ligadura tubária & V2 & $\begin{array}{l}\text { Ganho ponderal durante } \\
\text { a vida adulta de } 10 \text { a } 20 \mathrm{~kg}\end{array}$ \\
\hline M1 & $\begin{array}{l}\text { Menopausa com menos } \\
\text { de } 48 \text { anos }\end{array}$ & V3 & $\begin{array}{l}\text { Ganho ponderal durante } \\
\text { a vida adulta } \geq 20 \mathrm{~kg}\end{array}$ \\
\hline
\end{tabular}


canismo de participação da escolaridade no uso da TRH ocorra por esta aumentar a busca do conhecimento sobre saúde, possibilitando avaliação mais adequada dos riscos e benefícios desta terapia.

A análise de correspondência mostrou que a cor é capaz de discriminar as usuárias das não-usuárias. A cor branca, também mostrouse associada ao uso da TRH em outros estudos 24,25 . Além da associação com melhores condições sócio-econômicas, essa observação, talvez, possa ser explicada parcialmente pela prescrição seletiva do uso da TRH com a finalidade de prevenir a osteoporose, que apresenta maior incidência entre mulheres brancas.

Muitos estudos apontam que as usuárias da TRH são mais jovens em relação às não-usuárias $11,12,24,25,26$ e que a menopausa em menor idade associa-se ao uso de TRH 9. Esta observação é compreensível, pois a procura da TRH ocorre principalmente em decorrência da apresentação de sintomas típicos da fase inicial da menopausa.

A freqüência habitual de consulta odontológica de rotina correlaciona-se com a utilização de outros serviços de saúde em geral 27 . Outros estudos também observaram que o uso de serviço médico é mais freqüente entre as usuárias da TRH do que entre as não-usuárias 11,26 . Neste estudo observou-se de modo especial uma relação da irregularidade de visitas odontológicas com o não uso da TRH.

A condição de não-usuária mostrou forte correlação com a presença de sobrepeso aos 20 anos que foi evidenciada, também, em outros estudos 10,11,14,15,22,23,24,25, e um maior ganho ponderal durante a vida adulta. $O$ peso para as mulheres exerce importante papel no bem-estar físico, mental e social, podendo a magnitude do ganho ponderal representar um menor cuidado com sua saúde. Alternativamente, há o fato das mulheres com maior peso apresentarem sintomas mais suaves no climatério, em razão do maior nível de estrogênio circulante, gerado pela conversão periférica de androstenediona para estrona no tecido adiposo. Como a presença de sintomas é a característica mais marcante na indução das mulheres à procura da TRH, aquelas com sobrepeso ou obesidade tenderiam a utilizar menos este tratamento.

Os indicadores de saúde e saúde reprodutiva caracterizaram apenas o grupo de não-usuárias. A história de diagnóstico médico, prévio à menopausa, de hipertensão, diabetes ou hipercolesterolemia mostrou-se relacionada ao nãouso da TRH. Outros autores observaram um viés de prescrição 28 , por parte dos médicos com tendência a excluir da prescrição dessa terapia as mulheres portadoras de diabetes 14,24,25 e hipertensão arterial 15,23,26, entre outras patologias.

As características femininas relacionadas ao uso de métodos anticoncepcionais exemplificam a capacidade da mulher em estabelecer cuidados com relação à saúde reprodutiva. Em conseqüência, algumas variáveis no período reprodutivo poderiam se correlacionar com os cuidados no período não reprodutivo, como o uso da TRH. Há trabalhos demonstrando associação entre a contracepção oral e a TRH $10,11,12,24$. Neste estudo, entretanto, essa prática não distinguiu usuárias de não-usuárias de TRH, talvez em decorrência da não caracterização do tempo de uso, continuidade e motivo do tratamento, o que não permitiu captar o conceito de adesão diária e opcional ao uso do contraceptivo. As características de não planejar o primeiro filho, iniciar o pré-natal tardiamente e ser multípara caracterizaram as nãousuárias da TRH. A literatura aponta divergências quanto à associação da paridade ao uso da TRH. Persson et al. 14 apontaram associação da multiparidade com o não-uso da TRH. Porém, em outros estudos a maior paridade associou-se ao maior uso da TRH 13, talvez demonstrando que em algumas populações o acesso ao sistema de saúde durante a gravidez e o parto proporciona às mulheres maior confiança $\mathrm{e}$ conforto em utilizar os serviços médicos para outros fins.

Em resumo, um conjunto de características prévias à menopausa, como comportamentos desfavoráveis à saúde, em conjunto com padrões inferiores de renda e escolaridade, maior freqüência de diagnóstico médico de doenças e menor cuidado com a vida reprodutiva, apresentaram-se conjuntamente no grupo de mulheres não-usuárias de TRH. Esse perfil contrasta com o grupo das usuárias dessa terapia, que possui um padrão oposto, mais favorável à saúde.

Recentemente, o ensaio clínico norte-americano, Women's Health Initiative 2 , foi interrompido após a detecção de um aumento no risco primário de doença coronariana com o uso de estrogênio combinado à progesterona. A proteção cardiovascular evidenciada nos estudos observacionais anteriores vem sendo interpretada como artefatual, em conseqüência de viés de auto-seleção 7,8. Ou seja, apesar das análises nesses estudos incorporarem ajustes para fatores de risco estabelecidos para doença cardiovascular, tais procedimentos foram insuficientes para anular as diferenças prognósticas existentes entre usuárias e não-usuárias de TRH.

Em termos médicos, espera-se que o período pós-menopausa passe a ser objeto de uma 
abordagem mais integral, possibilitando propostas alternativas de promoção da saúde com estratégias não centradas prioritariamente em adesão a medicamentos, afastando-se assim do modelo de medicalização ainda predominante.

$\mathrm{O}$ estudo apresentado contém algumas limitações. Primeiro, algumas características estudadas estão mais sujeitas a erro de informação, em razão da utilização de questionário autopreenchível. Entretanto, estimativas de confiabilidade disponíveis sugerem alta qualidade dos dados incluídos nestas análises. Por exemplo, a confiabilidade teste-reteste (coeficiente de correlação intraclasse) do peso relatado aos 20 anos foi de 0,94 ; no caso da história de diagnóstico médico de hipertensão arterial em mulheres, o coeficiente kappa foi de 0,88 29. Segundo, não estão disponíveis informações sobre as razões pelas quais as mulheres optaram pelo uso da TRH, os regimes específicos e a du- ração do uso. Terceiro, dispomos de um número relativamente reduzido de mulheres pósmenopáusicas entre as participantes do nosso estudo. Por outro lado, exemplificou-se aqui uma vantagem adicional da análise de correspondência: como técnica multivariada, permite o aproveitamento integral de observações em que uma ou mais covariáveis apresentam dados faltantes, diferentemente de outros modelos de regressão múltipla.

Em nosso estudo, a análise de correspondência mostrou-se adequada para identificação de dois perfis distintos de mulheres em relação ao uso da terapia de reposição hormonal. A utilização desta técnica deve ser estimulada em pesquisas na área da saúde, por exemplo, para caracterização de subpopulações alvo de intervenções preventivas, que não se limite a características ou fatores de risco isolados.

\section{Resumo}

Neste estudo ilustra-se a utilização da técnica de análise de correspondência na caracterização do perfil das usuárias e das não-usuárias da Terapia de Reposição Hormonal (TRH). Analisou-se dados seccionais de 195 mulheres com relato de menopausa natural, entre 2.240 participantes do Estudo Pró-Saúde (Rio de Janeiro). As mulheres não-usuárias da TRH apresentaram perfil mais desfavorável em relação às usuárias dessa terapia, por exemplo, ganho ponderal mais acentuado na vida adulta, idade mais avançada, menopausa mais tardia e menor nível de escolaridade. $O$ uso desta ferramenta analítica deveria ser estimulada na área da saúde, em análises exploratórias das interrelações entre um grande número de variáveis em populações de interesse.

Terapia de Reposição Hormonal; Viés de Seleção; Estudos Longitudinais

\section{Colaboradores}

R. N. Aranha, E, Faerstein e G. Werneck participaram das etapas de análise e interpretação dos dados, e redação do artigo. G. M. Azevedo e C. S. Lopes contribuíram na redação do artigo. 


\section{Referências}

1. Beral V, Banks E, Reeves G. Evidence from randomised trials on the long-term effects of hormone replacement therapy. Lancet 2002; 360: 942-4.

2. Women's Health Initiative. Risks and benefits of estrogen plus progestin in healhty postmenopausal women. JAMA 2002; 288:321-33.

3. Stampfer MJ, Colditz GA. Estrogen replacement therapy and coronary heart disease: a quantitative assessment of the epidemiologic evidence. Prev Med 1991; 20:47-63.

4. Hulley S, Grady D, Bush T. Heart and estrogen/ progestin replacement study (hers) research group. randomized trial of estrogen plus progestin for secondary prevention of coronary heart disease in postmenopausal women. JAMA 1998; 280:605-14.

5. Humphrey LL, Chan BKS, Sox HC. Postmenopausal hormone replacement therapy and primary prevention of cardiovascular disease. Ann Intern Med 2002; 137:273-89.

6. Nelson HD, Humphrey LL, Nygren P, Teutsch SM, Allan JD. Postmenopausal hormone replacement therapy: scientific review. JAMA 2002; 288:872-81.

7. Grodstein F. Can selection bias explain the cardiovascular benefits of estrogen replacement therapy? Am J Epidemiol 1996; 143:979-82.

8. Rosenberg I. Hormone replacement therapy: the need for reconsideration. Am J Public Health 1993; 83:1670-3.

9. Chiechi LM, Berardesca C, Lobascio A, Carrieri M, Loizzi P. Postmenopausal users of long-term hormonal replacement therapy: social-cultural features. Clin Exp Obstet Gynecol 1999; 26:88-90.

10. Egeland GM, Kuller LH, Matthews KA, Kelsey SF Cauley J, Guzick D. Premenopeusal determinants of menopausal estrogen use. Prev Med 1991; 20: 343-9.

11. Hemminki E, Malin M, Topo P. Selection to postmenopausal therapy by women's characteristics. J Clin Epidemiol 1993; 46:211-9.

12. Isaacs AJ, Britton AR, McPherson K. Why do women doctors in the UK toke hormone replacement therapy? J Epidemiol Community Health 1997; 51:373-7.

13. Jensen LB, Hilden J. Sociological and behavioural characteristics of perimenopausal women with an express attitude to hormone substitution therapy. Maturitas 1996; 23:73-83.

14. Persson I, Bergkvist L, Lindgren C, Yuen J. Hormone replacement therapy and major risk factors for reproductive cancers, osteoporosis, and cardiovascular diseases: evidence of counfounding by expousure characteristics. J Clin Epidemiol 1997; 50:611-8.

15. Rodström K, Bengtsson C, Lissner L, Bjorkelund C. Pre-existing risk factor profiles in users and non-users of hormone replacement therapy: prospective cohort study in Gothenburg, Sweden. Br Med J 1999; 319:890-3.

16. Pinto Neto AMP, Pedro AO, Hardy E, Osis MJD, Costa-Paiva LHS, Martinez EZ. Caracterização das usuárias de terapia de reposição hormonal do Município de Campinas, São Paulo. Cad Saúde Pública 2002; 18:121-7.
17. Greenacre MJ. Correspondence analysis in pratice. London: Academic Press/Harcourt Brace \& Company; 1993.

18. Faerstein E, Lopes CS, Valente K, Solé Plá MA, Ferreira MB. Pré-testes de um questionário multidimensional autopreenchível: a experiência do Estudo Pró-Saúde UERJ. Physis (Rio J) 1999, 9: 117-30.

19. Fernandes CE, Pereira Filho AS. Manual de orientação de climatério. Comissão Nacional Especializada de Climatério. Rio de Janeiro: Federação Brasileira das Sociedades de Ginecologia e Obstetrícia; 1995.

20. Lebart L, Fénelon JP. Traitment des donees statistiques (Systeme Portable pour l'Ánalyse dês Donnés). http:/ www.linux.lettere.unige.it/htm/stat2/ 1.htm (acessado em 1/Fev/2003).

21. Chor D. Perfil de risco cardiovascular de funcionários de banco estatal [Tese de Doutorado]. São Paulo: Faculdade de Saúde Pública, Universidade de São Paulo; 1997.

22. Bush TL, Barret-Connor E, Cowan LD, Criqui MH, Wallace RB, Suchindran CM, et al. Cardiovascular mortality and non-contraceptive estrogen use in women: results from the Lipid Research Clinics'Program Follow-Up Study. Circulation 1987; 75:1102-9.

23. Matthews KA, Kuller LH, Wing RR, Meilahn EN, Plantinga P. Prior to use of estrogen replacement therapy, are users healthier than nonusers? Am J Epidemiol 1996; 143:971-8.

24. Nabulsi AA, Folsom AR, White A, Patsch W, Heiss $\mathrm{G}$, Wu KK, et al. Association of hormone-replacement therapy with various cardiovascular risk factors in postmenopausal womem. N Engl J Med 1993; 328:1069-75.

25. Keating NL, Cleary PD, Rossi AS, Zaslavsky AM, Ayanian JZ. Use of hormone replacement therapy by postmenopausal women in the United States. Ann Intern Med 1999; 130:545-53.

26. Buist DSM, Lacroix AZ, Newton KM, Keenan NL. Are long-term hormone replacement therapy users different from short-term and never users? Am J Epidemiol 1999; 149:275-81.

27. Hayward RA, Meetz HK. Utilization of dental service: patterns and trends. J Public Healht Dent 1989; 49:147-52.

28. Barret-Connor E. The menopause hormone replacement, and cardiovascular disease: the epidemiologic evidence. Maturitas 1996; 23:227-34.

29. Faerstein E, Chor D, Lopes CS. Reliability of the information about the history of diagnosis and treatment of hypertension. Differences in regard to sex, age, and educational level. The Pró-Saúde Study. Arq Bras Cardiol 2001; 76:297-304.

Recebido em 5/Set/2002

Versão final reapresentada em 9/Mai/2003 Aprovado em 2/Set/2003 